\title{
THE SOLUTION BY ITERATION OF NONLINEAR FUNCTIONAL EQUATIONS IN BANACH SPACES ${ }^{1}$
}

\author{
BY F. E. BROWDER AND W. V. PETRYSHYN
}

Communicated January 11, 1966

Introduction. Let $X$ be a Banach space, $T$ a (possibly) nonlinear mapping of $X$ into $X$. We are concerned with the solvability of the equation

$$
u-T u=f
$$

for a given element $f$ of $X$ and its relation to the properties of the Picard iterates for the Equation (1), i.e. the sequence $\left\{x_{n}\right\}$ where

$$
x_{n+1}=T x_{n}+f, \quad x_{0} \text { given. }
$$

In a preceding note on the linear case [8], we established the following facts for linear $T$ :

(a) If $X$ is reflexive and $T$ is asymptotically bounded (i.e. $\left\|T^{n}\right\| \leqq M$ for some constant $M$ and all $n \geqq 1$ ), then the Equation (1) has a solution $u$ for a given $f$ if and only if for any specific $x_{0}$, the sequence of Picard iterates $\left\{x_{n}\right\}$ starting with $x_{0}$ is bounded in $X$ (see [2]).

(b) For a general Banach space $X$, if $T$ is asymptotically convergent (i.e. $T^{n} x$ converges strongly in $X$ for each $x$ in $X$ as $n \rightarrow+\infty$ ), the sequence of Picard iterates $\left\{x_{n}\right\}$ for a given $x_{0}$ converges if and only if the equation (1) has a solution.

(c) For a general Banach space $X$ and $T$ asymptotically convergent, if an infinite subsequence of the sequence $\left\{x_{n}\right\}$ converges, then the whole sequence converges to a solution of Equation (1).

Our object in the present note is to give some partial extensions of these results to a general class of nonlinear operators $T$, and to indicate some interesting examples of the application of these nonlinear results.

THEOREM 1. Let $T$ be a nonexpansive nonlinear mapping of $X$ into $X$, (i.e. $\|T x-T y\| \leqq\|x-y\|$ for all $x$ and $y$ in $X$ ), and suppose that $X$ is uniformly convex. Then the Equation (1) has a solution u for a given $f$ in $X$ if and only if for any specific $x_{0}$ in $X$, the sequence of Picard iterates $\left\{x_{n}\right\}$ starting at $x_{0}$ is bounded in $X$.

Proof of Theorem 1. Let $T_{f}$ be the mapping of $X$ into $X$ given by $T_{f}(u)=T u+f$. Then $u$ is a solution of Equation (1) if and only if

${ }^{1}$ The preparation of this paper was partially supported by NSF Grant GP-3552. 
$u$ is a fixed point of $T_{f}$, and $T_{f}$ like $T$ is a nonexpansive self-mapping of $X$. If $T_{f}$ has such a fixed ponit $u$, then for each $n \geqq 1$,

$$
\left\|x_{n+1}-u\right\|=\left\|T_{f}\left(x_{n}\right)-T_{f}(u)\right\| \leqq\left\|x_{n}-u\right\| .
$$

Hence the sequence $\left\{x_{n}\right\}$ is bounded. The converse is a corollary, due to Belluce and Kirk [1], of the result established independently by Browder [6] and Kirk [9] that every nonexpansive self-mapping of a nonempty bounded close convex subset $C$ of a uniformly convex space has a fixed point. Indeed, let $d$ be the diameter of the set $x_{n}$, and for each $x$ in $X$, let $D_{d}(x)$ be the closed ball of radius $d$ about $x$. If $C_{k}=\bigcap_{j \geqq k} D_{d}\left(x_{j}\right), C_{k}$ is nonempty and convex for each $k$, and $T_{f}\left(C_{k}\right) \subset C_{k+1}$. Let $C$ be the closure of the union of $C_{k}$ for $k \geqq 1$. Since $C_{k}$ increases with $k, C$ is a closed bounded convex subset of $X$. Since $T_{f}$ maps $C$ into $C, T_{f}$ has a fixed point in $C$. q.e.d.

DEFINITION 1. The mapping $T$ is said to be asymptotically regular if for each $x$ in $X, T^{n+1} x-T^{n} x \rightarrow 0$ strongly in $X$ as $n \rightarrow+\infty$. T is said to be weakly asymptotically regular if $T^{n+1} x-T^{n} x \rightarrow 0$ weakly in $X$ as $n \rightarrow+\infty$ for each $x$ in $X$.

THeorem 2. Let $X$ be a Banach space, $T$ a nonexpansive mapping of $X$ into $X$. For a given $f$ in $X$, let $T_{f}(u)=T(u)+f$, and suppose that the mapping $T_{f}$ is weakly asymptotically regular. Let $x_{n}=T_{f}^{n} x_{0}$ be the sequence of Picard iterates for the Equation (1) starting with $x_{0}$, and suppose that an infinite subsequence of the sequence $\left\{x_{n}\right\}$ converges strongly to an element $y$ of $X$.

Then $y$ is a solution of Equation (1) and the whole sequence $\left\{x_{n}\right\}$ converges strongly to $y$.

Proof of Theorem 2. If $u$ is a solution of equation (1), i.e. a fixed point of $T_{f}$, then by Equation (3) above

$$
\left\|x_{n+1}-u\right\| \leqq\left\|x_{n}-u\right\| \text {. }
$$

If an infinite subsequence of $\left\{x_{n}\right\}$ converges to $u$, it follows that the whole sequence converges to $u$. Hence it suffices to show that the limit $y$ of the convergent subsequence $\left\{x_{n_{\pi}}\right\}$ of $\left\{x_{n}\right\}$ is indeed a fixed point of $T_{f}$.

By the assumption of weak asymptotic regularity of $T_{f}$, however, we know that

$$
\left(I-T_{f}\right)\left(x_{n}\right)=\left(I-T_{f}\right)\left(T_{f}^{n} x_{0}\right) \rightarrow 0
$$

weakly in $X$ as $n \rightarrow+\infty$. Since $x_{n_{k}} \rightarrow y$ strongly in $X$ and $\left(I-T_{f}\right)$ is continuous from $X$ to $X$ in the strong topology, $\left(I-T_{f}\right)\left(x_{n_{k}}\right)$ $\rightarrow\left(I-T_{f}\right)(y)$ strongly in $X$. Hence $\left(I-T_{f}\right)(y)=0$. q.e.d. 
Definition 2. The mapping $S$ of $X$ into $X$ is said to be demiclosed if for any sequence $\left\{u_{n}\right\}$ in $X$ with $u_{n} \rightarrow u$ weakly in $X, S u_{n} \rightarrow v$ strongly in $X$, we have $S u=v$.

THEOREM 3. Let $X$ be a Banach space, $T$ a nonexpansive mapping of $X$ into $X$ such that for a given $f$ in $X, T_{f}$ is asymptotically regular and $\left(I-T_{f}\right)$ is demiclosed. Let $F$ be the set of fixed points of $T_{f}$, and $\left\{x_{n}\right\}$ the sequence of Picard iterates for $T_{f}$ starting at $x_{0}$. Suppose that $T_{f}$ has at least one fixed point.

Then the weak limit of any weakly convergent subsequence of $\left\{x_{n}\right\}$ lies in $F$. In particular, if $X$ is reflexive and $F$ consists of exactly one point $y$, $\left\{x_{n}\right\}$ converges weakly to $y$.

Proof of Theorem 3. Suppose $F$ is nonempty and let $\left\{x_{n_{j}}\right\}$ be a weakly convergent subsequence of $\left\{x_{n}\right\}$ with weak limit $u$. By the asymptotic regularity of $T_{f},\left(I-T_{f}\right)\left(x_{n_{j}}\right) \rightarrow 0$ strongly in $X$. Since $T_{f}$ is demiclosed by hypothesis, it follows that $\left(I-T_{f}\right) u=0$, i.e. $u$ lies in $F$.

If $X$ is reflexive, each infinite subsequence of $\left\{x_{n}\right\}$ contains a weakly convergent subsequence whose limit lies in $F$. If $F$ consists of a single point, it follows that $x_{n}$ converges to that point weakly in $X$. q.e.d.

TheOREM 4. Let H be a Hilbert space, $T$ a nonexpansive self-mapping of $H$ such that $T_{f}$ is asymptotically regular for a given $f$ in $H$ and has a nonempty fixed point set $F$. Then the weak limit of any weakly convergent subsequence of $\left\{x_{n}\right\}$ lies in $F$. In particular, if $F$ consists of a single point $u$, then $x_{n}$ converges weakly to $u$.

More generally, these conclusions are valid for any Banach space $X$ having a weakly continuous duality mapping ([7]).

Proof of Theorem 4. It has been shown in Browder [4] using the theory of monotone operators in Hilbert space that if $T_{f}$ is a nonexpansive mapping, then $\left(I-T_{f}\right)$ is demiclosed. We then apply Theorem 3. (For further applications of the theory of monotone operators to the study of nonexpansive mappings, see Browder [3], [5].) The same conclusion is obtained for Banach spaces $X$ having a weakly continuous duality mapping $J$ (e.g. the spaces $l^{p}$ for $1<p$ $<+\infty)$ in Browder [7] using the theory of $J$-monotone operators. q.e.d.

TheOREM 5. Let $X$ be a uniformly convex Banach space, $T$ a nonexpansive self-mapping of $X$ with a nonempty set $F$ of fixed points. For a given constant $\lambda$ with $0<\lambda<1$, let $S_{\lambda}=\lambda I+(1-\lambda) T$.

Then $S_{\lambda}$ is asymptotically regular and has the same fixed points as $T$. 
Hence the fixed points of $T$ can be obtained from iteration of $S_{\lambda}$, for which the conclusions of Theorems 1-4 can be applied.

Proof of Theorem 5. It is obvious that the fixed point sets of $T$ and $S_{\lambda}$ coincide and that $S_{\lambda}$ is also a nonexpansive self-mapping of $X$.

Let $u$ be a fixed point of $T$, and for a given $x$ in $X$, let $x_{n}=S_{\lambda}^{n} x$. Since $S_{\lambda}$ is nonexpansive and $u$ is a fixed point of $S_{\lambda}$, it follows that $\left\|x_{n+1}-u\right\| \leqq\left\|x_{n}-u\right\|$ for all $n$, and hence that $\left\|x_{n}-u\right\|$ converges to a nonnegative limit $d_{0}$. Suppose that $d_{0}>0$. Since

$$
x_{n+1}-u=S_{\lambda}\left(x_{n}\right)-u=\lambda\left(x_{n}-u\right)+(1-\lambda)\left(T x_{n}-u\right)
$$

and since

$$
\left\|x_{n}-u\right\| \rightarrow d_{0}, \quad\left\|x_{n+1}-u\right\| \rightarrow d_{0}, \quad\left\|T x_{n}-u\right\| \leqq\left\|x_{n}-u\right\|,
$$

it follows from the uniform convexity of $X$ that

$$
\left\|\left(x_{n}-u\right)-\left(T x_{n}-u\right)\right\| \rightarrow 0,
$$

i.e. $x_{n}-T x_{n} \rightarrow 0$ strongly in $X$. Hence $x_{n+1}-x_{n} \rightarrow 0$ strongly in $X$, i.e. $S_{\lambda}$ is asymptotically regular. q.e.d.

REMARK. For compact nonexpansive mappings $T$, the mapping $S_{\lambda}$ with $\lambda=1 / 2$ and its iterates were first studied by Krasnoselskir [10]. For general $\lambda$, these mappings have been studied for compact $T$ by Schaefer [12] and for demicompact $T$ by Petryshyn [11]. All these results follow from the following:

Theorem 6. Let $X$ be a Banach space, $T$ a nonexpansive mapping of $X$ into $X$ which is asymptotically regular. Suppose that the fixed point set $F$ of $T$ is nonempty and that $(I-T)$ maps bounded closed subsets of $X$ into closed subsets of $X$.

Then for each $x_{0}$ in $X$, the sequence $T^{n} x_{0}$ converges strongly in $X$ to a fixed point of $T$.

Proof of Theorem 6. If $u$ is a fixed point of $T$, $\left\|T^{n} x_{0}-u\right\|$ does not increase with $n$. It suffices therefore to show that there exists a subsequence of $T^{n} x_{0}$ which converges strongly to a fixed point of $T$. Let $G$ be the strong closure of the set $\left\{T^{n} x_{0}\right\}$. By the asymptotic regularity of $T,(I-T)\left(T^{n} x_{0}\right)$ converges strongly to 0 as $n \rightarrow+\infty$. Hence 0 lies in the strong closure of $(I-T)(G)$, and since the latter is closed by hypothesis since $G$ is closed and bounded, 0 lies in $(I-T)(G)$. Hence there exists a strongly convergent subsequence of $\left\{T^{n} x_{0}\right\}$ which converges to an element $v$ of $G$ such that $(I-T) v=0$, i.e. $v$ is a fixed point of T. q.e.d.

REMARK. The hypothesis that $(I-T)$ maps bounded closed subsets 
of $X$ into closed subsets of $X$ is equivalent to the demicompactness of Petryshyn [11]. It is a consequence in particular of the stronger assumption that $T$ is compact, i.e. that $T$ maps bounded subsets of $X$ into precompact subsets of $X$.

\section{BIBLIOGRAPHY}

1. L. P. Belluce and W. A. Kirk, Fixed point theorems for families of contraction mappings, (to appear).

2. F. E. Browder, On the iteration of transformations in noncompact minimal dynamical systems, Proc. Amer. Math. Soc. 9 (1958), 773-780.

3. - Existence of periodic solutions for nonlinear equations of evolution, Proc. Nat. Acad. Sci. U.S.A. 53 (1965), 1100-1103.

4. - Fixed point theorems for noncompact mappings in Hilbert Space, Proc. Nat. Acad. Sci. U.S.A. 53 (1965), 1272-1276.

5. - Mapping theorems for noncompact nonlinear operators in Banach spaces, Proc. Nat. Acad. Sci. U.S.A. 54 (1965), 337-342.

6. - Nonexpansive nonlinear operators in a Banach space, Proc. Nat. Acad. Sci. U.S.A. 54 (1965), 1041-1044.

7. - Fixed point theorems for nonlinear semicontractive mappings in Banach spaces, Arch. Rational Mech. Anal. (to appear).

8. F. E. Browder and W. V. Petryshyn, The solution by iteration of linear functional equations in Banach spaces, Bull. Amer. Math. Soc. 72 (1966), 566-570.

9. W. A. Kirk, $A$ fixed point theorem for mappings which do not increase distance, Amer. Math. Monthly 72 (1965), 1004-1006.

10. M. A. Krasnoselskil, Two remarks about the method of successive approximations, Uspehi Mat. Nauk 10 (1955), no. 1 (63), 123-127.

11. W. V. Petryshyn, On the construction of fixed points and solutions of nonlinear equations with demicompact mappings, (to appear).

12. H. Schaefer, Über die Methode sukzessiver Approximationen, Jber. Deutsch. Math.-Verein. 59 (1957), 131-140.

University of Chicago 